\title{
Acro-osteolysis prior to diagnosis of leprosy
}

\author{
XIMENA ILLARRAMENDI, MÁRCIA R JARDIM*, \\ ANA M. SALES, JOSÉ A.C. NERY AND \\ EUZENIR N. SARNO \\ Leprosy Laboratory, Oswaldo Cruz Institute, Rio de Janeiro, Brazil \\ *Pedro Ernesto Hospital, Rio de Janeiro State University, Brazil
}

\author{
Accepted for publication 9 June 2000
}

Summary Acro-osteolysis (bone resorption) has been observed in a heterogeneous group of congenital and acquired bone disorders. Leprosy is the main cause of peripheral neuropathy leading to acro-osteolysis in endemic countries. Pure neuritic leprosy, a less common form of the disease, is difficult to diagnose. Two unrelated leprosy patients with acropathy whose disease began as pure neuritic are discussed.

\section{Introduction}

Acro-osteolysis is part of a heterogeneous group of disorders in which progressive skeletal rarefaction leads to disappearance of one or more bone segments of the distal part of the extremities, i.e. the phalanges, or the tarsocarpal bones. ${ }^{1,2}$ Spontaneous progressive idiopathic bone resorption has been described with minimal soft tissue loss and may be associated with additional systemic manifestations. ${ }^{1}$ Acquired or secondary acro-osteolysis may be due to metabolic disease, peripheral neuropathy, exposure to chemicals, or may result from inflammatory and vascular processes. ${ }^{1,3}$ Leprosy is an important cause of acral bone resorption.

Impairment more frequently occurs towards the lepromatous end of the spectrum of leprosy ${ }^{4}$ and may occur at any stage of the disease. Mutilation, defined as the partial or total loss of a segment, ${ }^{5}$ represents the last stage of deformity; it disables the individual for simple activities and increases the stigma carried by the disease even more. Mutilations are not as common today due to rigorous implementation of control programmes, leading to case finding and better management of the disease. Early detection of nerve damage is essential for the prevention of disability in leprosy. ${ }^{6}$ Pure neuritic leprosy is more difficult to diagnose due to the absence of skin lesions as well as acid-fast bacilli (AFB) in slit-skin smear. Hence, for many patients with pure neuritic disease the disease progressed without treatment.

Correspondence to: Dr Euzenir Nunes Sarno, Laboratório de Hanseníase, Instituto Oswaldo Cruz, Av. Brasil 4365, CEP 21045-900, Rio de Janeiro, RJ, Brasil (e-mail: esarno@gene.dbbm.fiocruz.br) 


\section{Case reports}

CASE 1

A.C.F. was a male, 39 years of age, Caucasian, native from Rio de Janeiro, illiterate and supported by welfare benefits. He was apparently healthy until November 1982, when he developed a post-traumatic vesicular lesion on the second left toe that became ulcerated and would not heal. During ambulatory follow-up, Raynaud's phenomenon, 'mal perforant du pied', painless oedema of ipsilateral great toe, alteration of sensation on extremities, plantar and elbow hyperkeratosis and multiple scars on the extremities were observed. No leprosy skin lesions were found. Neurological evaluation revealed loss of pain and temperature sensation with preservation of light touch in glove and stocking distribution, together with thickening of several nerves. Biopsies of normal skin and sural nerve showed non-specific inflammation and chronic neuritis, respectively (see Figure 1). Biopsy of bone was compatible with neurotrophic arthropathy. In later evaluations, thinning of both fifth fingers and mild shortening of the second right finger, of fourth right toe, and of both great toes was observed. Due to persistence of signs and the development of hepato-splenomegaly he was hospitalized on three occasions $(1983,1984,1985)$. Over the next 5 years, he was followed without having a definitive diagnosis. Various aetiologies were considered, such as primary amyloidosis, bone tumour, leprosy and hereditary sensory and autonomic neuropathies, the latter considered to be the most likely diagnosis.

Five years later, a hypochromic hypoaesthetic macula was observed on the patient's back. The skin smear was negative but a skin lesion biopsy revealed AFB; leprosy was therefore the final diagnosis. By that time, severe deformities had developed in the form of bilateral clawing, moderate shortening of fingers and toes and chronic plantar ulcers. Treatment with MDT according to World Health Organization standards (MDT/WHO) was started on December of 1987.

In 1990 he was released from MDT/WHO with disability grade 2, but was kept under surveillance by physiotherapy and dermatology. No reactive states were diagnosed during treatment or at follow-up. Alteration of sensation persisted. He suffered from chronic plantar

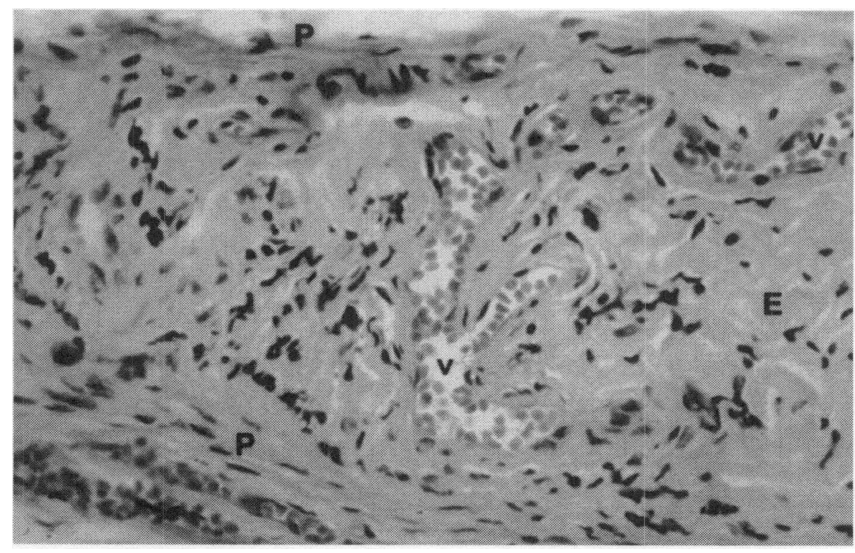

Figure 1. Case 1. Nerve histology (sural nerve, haematoxylin eosin, $\times 400$ ) showing inflammatory cells and neovascularization (v) within the endoneurium (E). Fibrotic tissue is observed in substitution of nerve fibres both in the (E) and the perineurium $(\mathrm{P})$. 


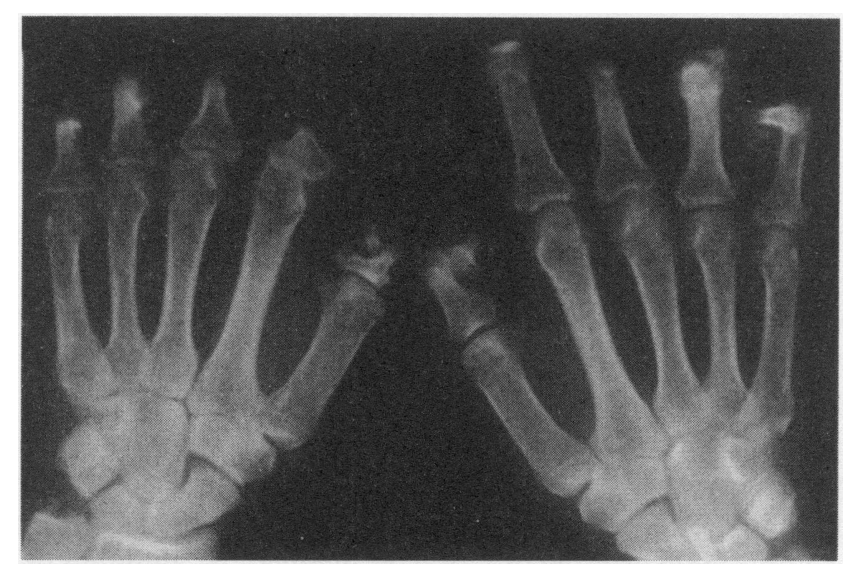

Figure 2. Case 1. Anteroposterior X-ray of hands showing various patterns of bone resorption: lateral, frontal and distal osteolysis with fragmentation. Also subluxation of 1st, 4th and 5th distal interphalangeal articulations.

ulcers and successive episodes of osteomyelitis, which led to amputation of two fingers and continuous extrusion of sequestra. Chronic Achilles tendonitis and severe acral deformities developed (see Figure 2).

\section{CASE 2}

M.F.S.B. was a female, 57 years of age, widow, seamstress and native of the State of Paraiba. She was referred to our outpatient clinic after local anaesthesia was observed following an extensive burn on the left forearm. She reported developing illness 10 years earlier with right foot anaesthesia. She sought medical assistance because of right foot drop and development of an ulcer on the fourth right toe. At that time, no investigations were done. She observed progressive shortening of the 4th right toe and development of a chronic plantar ulcer under the 4 th and 5th metatarsals. The patient reported no family history of similar disease or alcohol habit.

Palmar and plantar erythema and cyanosis, right fibular nerve thickening, ipsilateral foot drop, and severe shortening of 4th and 5th right toes were observed upon admission. No leprous skin lesions were observed. Examination of sensation revealed tactile, thermal and pain anaesthesia on the topography of medial and lateral cutaneous nerves of left forearm, together with sural, fibular, saphenous, calcaneous and lateral and medial right plantar nerves. There was tactile hypoaesthesia in the distal region of the left lower extremity. Reduction in muscular force of the distal right lower extremity and of intrinsic left hand muscles was also observed. Slit-skin smears were negative for AFB. Electromyography was performed and showed lack of response of right sural and fibular nerves, with axon damage in the median, ulnar and left fibular nerves, compatible with mononeuropathy multiplex (see Figure 3). A biopsy of the right sural nerve demonstrated total fibrosis without signs of inflammatory infiltrate. Treatment for pure neuritic leprosy with multidrug therapy for paucibacillary patients $(600 \mathrm{mg}$ of monthly supervised rifampicin and $100 \mathrm{mg}$ of daily dapsone) was initiated. She developed a plantar ulcer on the right foot, which healed after physiotherapy and care. 

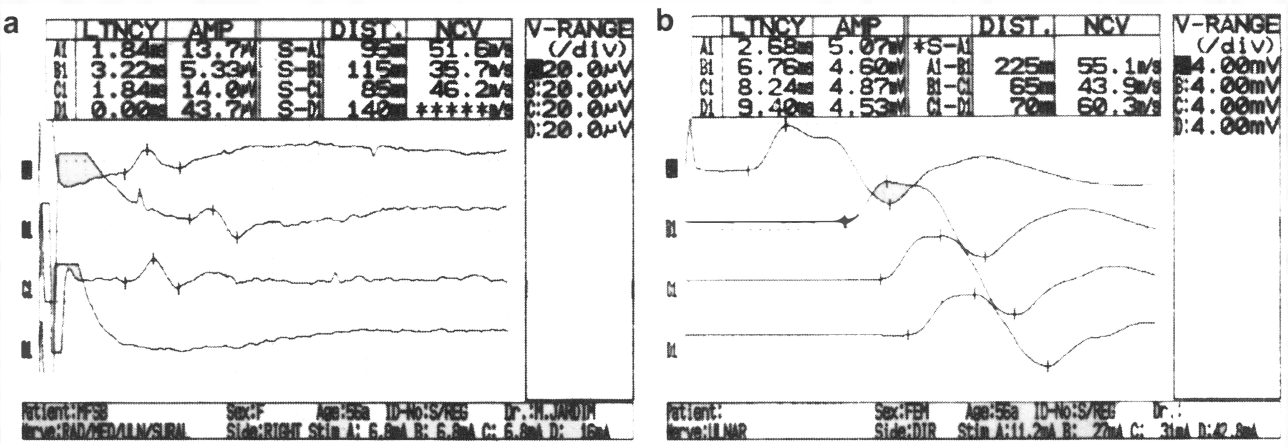

Figure 3. Case 2. Nerve conduction study compatible with mononeuropathy multiplex. (a) Sensory conduction of ulnar, radial and sural nerves shows lack of response of right sural nerve with axon damage in the median, ulnar, and left fibular nerves. (b) Motor conduction of right ulnar nerve. There is a reduction in conduction velocity and axonal damage of the nerve below the elbow.

\section{Discussion}

Neuropathy in the absence of skin lesions, known as 'pure neuritic' or 'primary neural' leprosy, is considered rare. Kundu et al. ${ }^{7}$ reported a frequency of 34 in 11,000 cases of leprosy in Calcutta, while other authors report a global prevalence of $45-5 \%{ }^{8,9}$ However, with electrophysiological evaluation and nerve biopsies, a higher prevalence is reported nowadays. In our Brazilian practice during the last 5 years, we have seen 34 cases of pure neuritic leprosy among a total of 635 patients. We have observed an increase in the detection of leprosy cases that are pure neuritic, from $192 \%$ in 1994 to $1053 \%$ during 1999.

Any neurological disease that results in loss of pain sensation in extremities without paralysis of the affected segment may result in bone resorption. Pandya ${ }^{10}$ described the acrodystrophic neuropathies as a heterogeneous group of acquired and hereditary diseases of sensitive fibres in which ulceration of palms and soles were a prominent sign. In addition to neuropathic acro-osteolysis, primary and other acquired causes have been described ${ }^{1-3}$ and must be considered in the differential diagnosis.

Hereditary sensory and autonomic neuropathy type $I$ is a dominantly inherited ulcerative-mutilating acropathy with insidious onset of symptoms in the second or later decades of life. ${ }^{11,12}$ There is progressive sensory and autonomic loss over feet and distal aspects of legs with no or slight involvement of acral parts of upper limbs. Although case 1 reported no family history of similar illness, it remained as the diagnosis of his disease due to the similarity in progression ${ }^{13}$ until the bacillus was found. This uncommon neuropathy is to be considered as a diagnosis of exclusion. Instead, leprosy should be the most probable diagnosis in an endemic region, in the knowledge that slit-skin smear is negative for AFB in all patients with neuritic leprosy and that nerve histology can be inconclusive. ${ }^{14,15}$ The characteristic features of electromyography in leprosy may facilitate the diagnosis; however, in many areas in endemic countries performing this study may not be possible.

In the two cases presented here, the difficulties encountered in diagnosing pure neuritic leprosy at an early stage permitted the disease to develop unchecked for several years, without receiving specific treatment. Even in endemic areas in which the clinical 
features of thickened nerves accompanied by nerve deficit are known to reflect neuritic leprosy, accurate diagnosis remains hard to come by and it is often delayed if diagnosed at all. As a result, there are greater opportunities for these patients to develop deformities and mutilations especially if we take into consideration that both clinical and electrophysiological abnormalities positively correlate to the duration of symptoms of leprosy. ${ }^{6}$

Various epidemiological factors such as age, occupation, gender and form of leprosy have been reported to contribute to the development of disabilities and deformities. ${ }^{16}$ Pure neuritic leprosy, either due to the difficulty in diagnosis or to the delay of patient's perception of loss of sensation, especially among illiterate individuals, allows the insidious evolution of the disease to occur unchecked. Pure neural leprosy undoubtedly adds to the prevalence of deformities in Hansen's disease. However, whatever the cause of the peripheral nerve damage, appropriate teaching about self-care of anaesthetic areas can prevent much of the secondary deformity.

As stated above, deformities and mutilations have been observed in all forms of leprosy, even in the absence of clinical signs of infection or ulcers. It is our intention to draw special attention to the not-so-uncommon pure neuritic form of leprosy that is little diagnosed. For this reason, it is imperative that neurologists keep this cause of sensory neuropathy in mind and that leprosy clinics ensure that an experienced neurologist is readily available for consultation.

\section{Acknowledgements}

We are grateful to Ms Judith Grevan for the correction of the manuscript. We thank Dr H. Srinivasan for his counsel. These results were presented at the Third International Congress on the Evolution and Palaeoepidemiology of Infectious Diseases: Past and Present of Leprosy, Bradford University, UK, July 27, 1999.

\section{References}

1 Todd G, Saxe N. Idiopathic phalangeal osteolysis. Arch Dermatol, 1994; 130: 759-762.

2 Bisagni-Faure A, Giraudet-Le Quintrec JS, Job-Deslandre C, Menkes CJ. L'osteolyse essentielle. Ann Med Int, 1991; 142: 17-20.

${ }^{3}$ Kemp SS, Daliknka MK, Schumacher HR. Acro-osteolysis. Etiologic and radiological considerations. JAMA, 1986; 255: 2058-2061.

4 Smith WCS. The epidemiology of disability in leprosy including risk factors. Lepr Rev, 1992; 63: 23S-30S.

${ }_{5}^{5}$ Mallac MJ. Onset and pattern of deformity in leprosy. Lepr Rev, 1966; 37: 71-91.

${ }^{6}$ Brown TR, Kovindha A, Wathanadilokkol U et al. Leprosy neuropathy: correlation of clinical and electrophysiological tests. Ind J Lepr, 1996; 68: 1-14.

7 Kundu KK, Ghosh S. Observations on clinical manifestations of primary polyneuritic type of leprosy. Ind $J$ Dermatol, 1970; 15: 45-48. Quoted in Gadoth N, Bechar M, Kushnir M, Davidovitz SU. Somatosensory and autonomic neuropathy as the only manifestation of long stantding leprosy. J Neurol Sci, 1979; 43: 471-477.

${ }^{8}$ Mahajan PM, Jogaikar DG, Mehta JM. A study of pure neuritic leprosy: clinical experience. Ind J Lepr, 1996; 68: $137-141$.

9 Girdhar BK. Neuritic leprosy. Ind J Lepr, 1966; 68: 35-42.

${ }^{10}$ Pandya SS, Chulawala RG. Electrophysiologic and histologic studies in leprosy and some acrodystrophic neuropathies. Int J Lepr, 1981; 49: 398-405.

11 Allmann K-H, Leu H, Burg G, Hodler J. Hereditary sensory and autonomic neuropathy I (Thévenard's disease). Skeletal Rad, 1996; 25: 501-504.

12 Pavithran K. Hereditary sensory neuropathy type-I simulating pure neuritic leprosy. Ind J Lepr, 1992; 64: 201-203. 
13 Dyck PJ. Neuronal atrophy and degeneration predominantly affecting peripheral sensory and autonomic neurons. In: Dyck PJ, Thomas PK, Lambert EH, Bunge R (eds) Peripheral neuropathy, vol II, Chapter 68. Saunders, USA, p 1561.

14 De Freitas MRG, Nascimento OJM, Drago MJ et al. Paralisia do nervo ulnar na lepra sem alterações cutâneas. Biópsia do ramo superficial do nervo ulnar na mão. Arq Neuropsiquiatria, 1998; 56: 585-594.

15 Kaur G, Girdhar BK, Girdhar A et al. A clinical, immunological and histological study of neuritic leprosy patients. Int J Lepr, 1991; 59: 385-391.

16 Thappa DM, Sharma VK, Kaur G. Disability index of hands and feet in patients attending an urban leprosy clinic. Ind J Lepr, 1990; 62: 328-337. 